\title{
PREVENTION \\ OVERVIEW OF MEDICAL MANAGEMENT OF VICTIMS OF SEXUAL ABUSE
}

A H Wulfsohn, $M B B C h$

Head, Sunninghill Hospital Accident and Emergency Unit, and Medical Director, Albertina Sisulu Rape Crisis Centre, Johannesburg

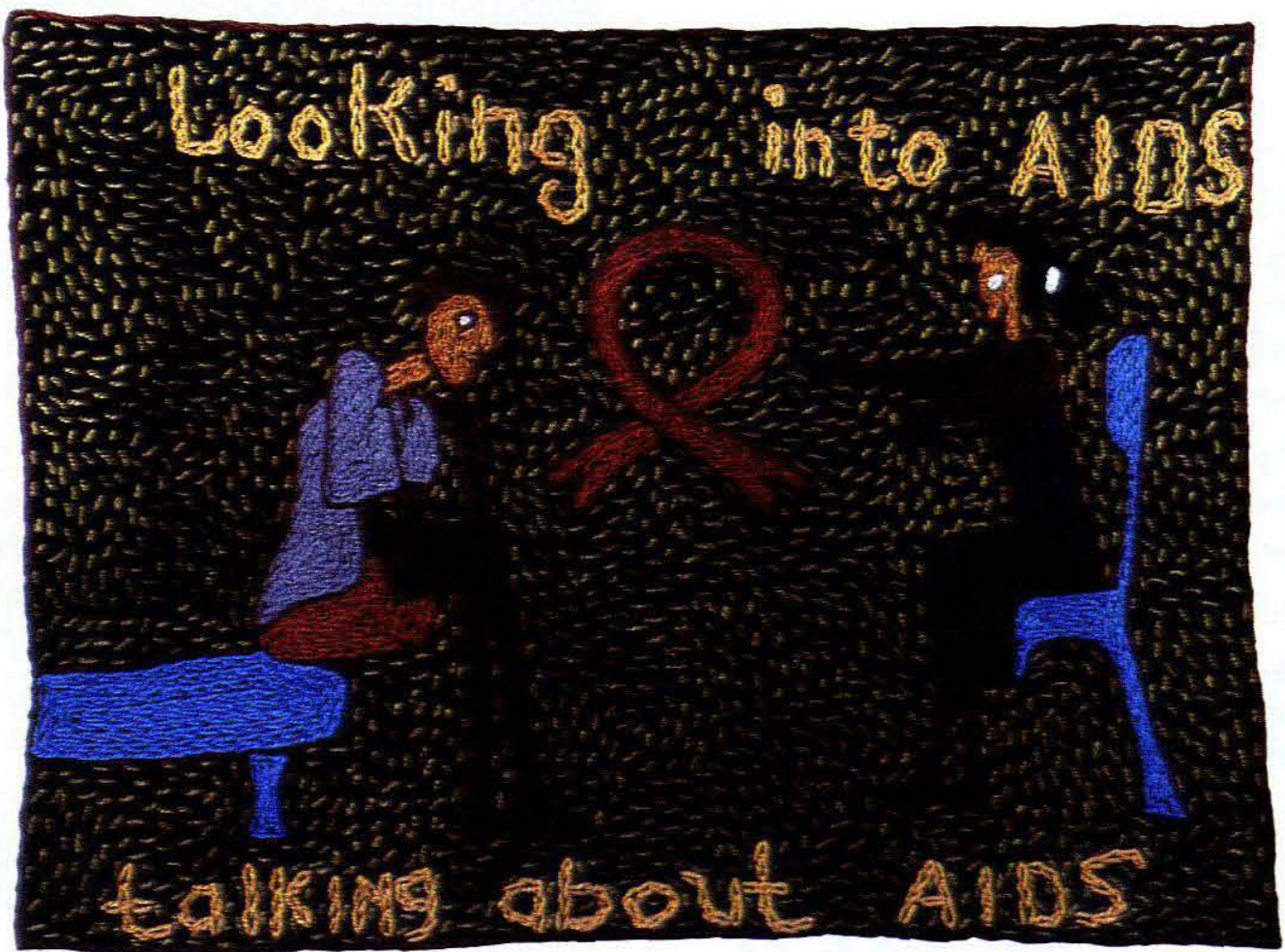

As general medical practitioners, few of us have seen and managed rape victims on a regular basis. A major reason for this is that until recently only district surgeons saw and treated rape victims. However, if one takes the time to read the various provincial Health Departments' medico-legal services guidelines, all experienced registered health care workers may see and manage these 'types of patients'.

A sexual assault occurs once every 6.4 minutes in the USA, where 1 in every 6 women will be raped during her lifetime. Although a woman is four times more likely to be assaulted by someone she knows than by someone she does not know, the majority of these crimes go unreported, even though rape is seen to be a felony in the United States. ${ }^{1,2}$

Statistics from UNISA (March 1999) show that one in two South African women will be raped. ${ }^{3}$ In 1999 Childline showed that 1 in 4 girls and 1 in 5 boys under the age of 16 years will have been sexually molested. ${ }^{3}$ In May 2000 the Department of Health showed that $20 \%$ of girls aged between 13 and 19 years and seen at antenatal clinics were infected with HIV. The various statistics are endless and horrifying - as medical professionals what are we doing to alleviate the problem?

Unfortunately, it took a tragic incident in which a 15year-old girl was gang-raped after the intruders had murdered both her parents, for me to realise how inadequate the system was.

In March 1998 the Sunninghill Hospital Rape Crisis Centre was opened. After extensive literature research and discussions with experts nationally and internationally, basic protocols and guidelines were drawn up.

If taken within 2 hours of occupational exposure, post-exposure prophylaxis (PEP) reduces the risk of seroconversion by approximately $80 \%$. It is now recommended that health care workers take PEP within 72 hours following needlestick injury. No reliable data exist for PEP in rape victims, but trials done in San Francisco ${ }^{4}$ and France $^{5}$ suggest that effectiveness may be as high as 100\% if PEP is taken within 72 hours of the incident. 
Management of a rape survivor can be conveniently regarded under the following headings, which comprise guidelines used at Sunninghill Hospital and the other sexual assault crisis centre clinic within Netcare:

\section{MEDICAL MANAGEMENT AT SUNNINGHILL} HOSPITAL, JOHANNESBURG

It is important to remember that a rape survivor is a patient like any other, though with special needs. Therefore, the basics of clinical medicine apply: good history taking, clinical examination, special investigations and appropriate management.

1. Every patient is seen and managed in a comfortable and compassionate environment.

2. Should the patient be a priority 1 (code red immediate life-threatening injury) or a priority 2 (code yellow -limb/potential life-threatening injury), then the patient is managed in the resuscitation room according to advanced trauma life support protocols.

3. If the patient is a priority 3 (code green - non-limb or life-threatening injury) then the patient is treated in a private dedicated room.

4. A registered nurse and a medical doctor start taking a medical history as well as details of the actual rape.

5. All staff have undergone basic counselling training and have been trained in general medico-legal procedures.

6. Once a history has been taken the procedures are explained in detail to the patient.

7. If the patient has decided to lay a charge the appropriate medico-legal examination will take place.

8. At this and other similar units the appropriate crime kits for the medico-legal examination are available. Often the investigating South African Police officers may not have easy access to the specific kits.

9. For this reason a co-operative positive working relationship with the relevant police officers is vital.

10. All injuries are attended to within the unit. The patient should not be transferred unless procedures not routinely provided for in the unit are necessary.

11. Specialist treatment may be required, e.g. a child with vaginal tears should be sutured under general anaesthetic administered by a specialist anaesthetist.

12. If the patient requires $X$-rays, admission to hospital or other specific medical treatment will be done. This alleviates any further secondary traumatisation for the patient.
13. Antibiotic prophylaxis is prescribed where necessary.

14. Following completion of the full medico-legal examination, a facility where the patient can shower/bath and change into clean clothing should be made available.

15. The patient is followed up at 6-weekly, 3-monthly and 6-monthly intervals and annual intervals.

16. The patient is free to attend the unit for any ongoing medical condition or counselling and for regular annual follow-up visits.

\section{POST-EXPOSURE PROPHYLAXIS (PEP)}

1. Part of the initial history-taking involves pre-HIV test counselling.

2. Information regarding PEP guidelines is given to the patient.

3. Informed consent for the baseline HIV ELISA test is obtained.

4. Information is given and consent obtained with regard to further management.

5. If the patient is under age, then consent should be provided by the appropriate parent/guardian. If they are not available, then attending police officers will assist.

6. A quick turnaround time is required for the HIV ELISA results which are returned within 3 hours by Ampath Laboratories.

7. Only patients who attend the unit within 72 hours of the incident will be offered PEP should the baseline ELISA test be negative.

8. If the baseline HIV ELISA is positive, counselling is carried out and referrals are made to appropriate centres for HIV-AIDS care.

9. Other baseline tests include: a full blood count (FBC), liver function (LF) and urea and creatinine estimation.

10. A pregnancy test will be performed if necessary.

11. The patient will be followed up by the unit for other medical problems as well as for ongoing counselling.

12. If the patient attends the unit 72 hours after the incident, counselling is carried out and the blood tests mentioned above are offered.

13. No PEP is offered to patients attending after 72 hours. ${ }^{3.46}$

14. All patients who present within 72 hours of the incident and are HIV-negative are offered a 28-day course of AZT $200 \mathrm{mg}$ bd and 3TC $150 \mathrm{mg}$ tds.

15. Of the $>450$ patients who have been seen at the Sunninghill Hospital Rape Crisis Centre, only 2 
patients have been given indinavir sulphate (Crixovan). (One of the patients was a health care worker who requested the therapy, and the second was a code red assault.)

\section{RESULTS TO DATE}

1. Approximately $18 \%$ of patients attending the unit are HIV-positive.

2. Of the patients who were HIV-negative at baseline (this is the majority of patients) and who took PEP within 72 hours of the rape and completed the course, none has seroconverted.

3. In one case, PEP was given more than 72 hours after the incident (supplied by an external source). This patient tested positive at the 6 -week visit. (This patient was negative at baseline; however, she was tested 4 days after the incident.)

4. Severe side-effects were rare and only noted in 2 patients.

5. Compliance is not a problem with PEP in the context of sexual assault whereas compliance for PEP in the case of needlestick injuries among health care workers has been problematic because of sideeffects.

6. The follow-up rate is satisfactory until the third month (approximately 68\%), but falls away dramatically after the 6-month visit to as low as 35\%.

\section{OTHER MEDICATIONS}

1. Where appropriate, the patient is also given antibiotic prophylaxis:

penicillin 2 million units imi OR $2 \mathrm{~g}$ ciprofloxacin stat

metronidazole $2 \mathrm{~g}$ po stat OR metronidazole $500 \mathrm{mg}$ ivi (should the patient be vomiting or nauseous)

tetracyclines 500 mg qid for 10 days.

2. Children allergic to penicillin are given erythromycin.

3. A 'morning after pill', e.g. levonorgestrel $0.25 \mathrm{mg}$ and ethinylestradiol $0.05 \mathrm{mg}$ or a higher dose norgestrel/ethinyl oestradiol combination (two tablets stat and then two 12 hours later - in both formulations mentioned the dosage is the same) is supplied if necessary.

4. Vaginal douches are supplied for patients to take home.

5. Anti-emetics are prescribed if necessary.

6. Analgesia/anti-inflammatory agents are also provided if necessary.

\section{COUNSELLING}

1. Trained counsellors are called in to counsel every rape patient. Support is provided to the family, friends, spouse and patient during the acute event and follow-up counselling, tailored to the patient's needs, is provided on a regular basis.

2. The unit's experience is that it is imperative that a counsellor see the patients at their first visit. This facilitates regular follow-up for the patients and their families.

3. Should patients wish to lay a charge, they may do so with the attending police officer. (Police officers accompany less than $48 \%$ of the patients who come to the unit; however, the unit's figures show that over $70 \%$ of patients actually lay charges.)

4. If a police officer is not in attendance during the initial admission then the appropriate police services must be called should the patient wish to lay a charge.

When formulating guidelines and protocols for this disastrous medical emergency it is important for medical practitioners to remember that every patient is an individual and that management of the patient and family needs to be individualised. It is also necessary to bear in mind that both the physical and emotional injuries may need therapy for several months and ongoing support will need to be provided for a considerable period of time.

In common with PEP guidelines in other settings, it is recognised that in this context revision may need to occur as and when new therapies and new testing methods become available.

\section{REFERENCES}

1. Petter $L M$, Whitehill DL. Management of female sexual assault. Am Fom Physicion 1998.

2. Statistical Abstract of the United States 1996, 116 th ed.

3. Swart L Gilchrist A, Butchard A, Seedat M, Martin L Rape Surveillance Through District Surgeon's Offices in Johannesburg, 1996 - 1998: Evaluation and Prevention Implications. Pretoria: Institute of Social and Health Sciences, University of South Africa, March 1999.

4. Bamberger JD, Waldo CR, et al, Post exposure prophylaxis for HIV infection following sexual assault. Am J Med 1999; 106: 323-326.

5. Beinot JP, et al. An investigation of rape and post exposure prophylaxis in eight Parisian clinics. Paper presented at the Durban World AIDS Conference, June 2000.

6. Gerberding $J$. Prophylaxis for occupational exposure to HIV. Ann Intern Med 1996: 125: 497-501.

FURTHER READING

- Case-control study of HIV seroconversion in health-care workers after percutaneous exposure to HIV infected blood: France, UK, and USA, January 1988 - August 1994. MMWR 1995; 44: 929-933.

- Centres for Disease Control and Prevention. Guidelines for treatment of sexually transmitted diseases. MMWR 1998; 47: 109-111.

- Gostin LO, Lazzarini Z, Alexander D, et al. HIV testing, counseling, and prophylaxis after sexual assault. JAMA 1994; 271: 1436-1444.

- Katz MH, Gerberding JL. Post exposure treatment of people exposed to the HIV through sexual contact of injection-drug use. N Eng/ J Med 1997; 336: 1097-1100. 